\title{
A pore-forming protein drives macropinocytosis to facilitate toad water maintaining
}

\author{
Zhong Zhao ${ }^{1,2}$, Zhi-Hong Shi ${ }^{1,2}$, Chen-Jun Ye ${ }^{1}$, Yun Zhang ${ }^{1,3} *$
}

1. Key Laboratory of Animal Models and Human Disease Mechanisms of the Chinese Academy of Sciences/Key Laboratory of Bioactive Peptides of Yunnan Province, Kunming Institute of Zoology, the Chinese Academy of Sciences, Kunming, Yunnan 650223, China

2. Kunming College of Life Science, University of Chinese Academy of Sciences, Kunming, Yunnan 650204, China

3. Center for Excellence in Animal Evolution and Genetics, Chinese Academy of Sciences, Kunming, Yunnan 650223, China

\# These authors contributed equally to this work

* Author to whom correspondence should be addressed:

Dr. Yun Zhang

Kunming Institute of Zoology, Chinese Academy of Sciences

32 East Jiao Chang Road

Kunming 650223, Yunnan, China (PRC)

Tel: +86-871-65198515; Fax: +86-871-65191823; 65198515

E-mail: zhangy@mail.kiz.ac.cn 


\begin{abstract}
Maintaining water balance is a real challenge for amphibians in terrestrial environments. Our previous studies with toad Bombina maxima discovered a secretory aerolysin family pore-forming protein and trefoil factor complex $\beta \gamma$-CAT, which is assembled under tight regulation depending on environmental cues. Here we report an unexpected role for $\beta \gamma$-CAT in toad water maintaining. Deletion of toad skin secretions, in which $\beta \gamma$-CAT is a major component, increased animal mortality under hypertonic stress. $\beta \gamma$-CAT was constitutively expressed in toad osmoregulatory organs, which was inducible under the variation of osmotic conditions. The protein induced and participated in macropinocytosis in vivo and in vitro. During extracellular hyperosmosis, $\beta \gamma$-CAT stimulated macropinocytosis to facilitate water intake and enhanced exosomes release, which simultaneously regulated aquaporins distribution. Collectively, these findings uncovered that besides membrane integrated aquaporins, a secretory pore-forming protein can facilitate toad water maintaining via macropinocytosis induction and exocytosis modulation, especially in responses to osmotic stress.
\end{abstract}




\section{Introduction}

Maintaining water balance is a key challenge that amphibians face in the process of transition from water to land $(1,2)$. In these animals water storage and utilization are enhanced and evaporation is reduced by coordinated functions of the nervous, endocrine and lymphatic systems, and of organs involved in water and salt balance and osmotic regulation, including skin, urinary bladder (UB) and kidney $(1,3)$. Amphibians are able to absorb water through their skin $(1,2)$. In addition, water is reabsorbed from the tubular fluid in the kidney and from stored urine in the $\mathrm{UB}(2,3)$. Water channel proteins aquaporins (AQPs) play key roles in transepithelial water absorption/reabsorption in these organs, and in cell volume regulation $(4,5)$. However, it has rarely been studied in the physiological function of protein components from amphibian skin secretions in regulating of integumental water homeostasis.

Macropinocytosis is a mechanism that mediates bulk uptake and internalization of extracellular fluid and the solutes contained therein, producing endocytic vesicles with diameters of $0.2-5 \mu \mathrm{m}(6,7)$. Macropinocytosis is an actin-dependent endocytic pathway mediated by the activation of the Ras and phosphatidylinositol 3-kinase (PI3K)-signaling pathways, which is induced by either endogenous agents such as growth factors, or by invasive microbes $(6,8)$. This fundamental cellular process has been documented to play various patho-physiological roles in a range of normal and malignant cells, including nutrient acquisition, cell growth, traffic and renewal of membrane components, immune surveillance, entry of pathogens, and cellular motility $(6,9,10)$. However, the physiological roles of macropinocytosis, a very ancient form of endocytosis, remain incompletely understood $(11,12)$.

Pore-forming proteins (PFPs) are usually secretory proteins that exist in a water-soluble monomeric form and oligomerize to form transmembrane pores (channels) $(13,14)$. Aerolysins are bacterial $\beta$-barrel PFPs produced by Aeromonas species $(13,14)$. Interestingly, numerous aerolysin family PFPs (abbreviated af-PFPs, previously referred as Aerolysin-Like Proteins, ALPs) harboring an aerolysin membrane insertion domain fused with other domains have been identified in various 
animals and plants $(15,16)$. Our previous studies with the skin secretions of the toad Bombina maxima discovered an interaction network among af-PFPs and trefoil factors (TFFs) (17). BmALP1, an af-PFP from the toad, can be reversibly regulated between the active and inactive forms, in which its paralog BmALP3 is a negative regulator depending on environmental oxygen tension (18). Specifically, BmALP1 interacts with BmTFF3 to form a membrane active PFP complex named $\beta \gamma$-CAT (19-21), in which BmTFF3 acts as an extracellular chaperon that stabilizes the BmALP1 monomer and delivers this PFP to its proper membrane targets $(18,21,22)$.

This secretory PFP complex $\beta \gamma$-CAT targets gangliosides and sulfatides in cell membranes in a double-receptor binding model (22). Then the BmALP1 subunit is endocytosed and this PFP oligomerizes to form channels on endolysosomes, modulating the contents and biochemical properties of these intracellular organelles (22-25). Thus, $\beta \gamma$-CAT is a secretory endolysosome channel (SELC) protein, representing a hitherto unknown SELC pathway (17). Depending on cell contexts and surroundings, the cellular effects of SELC protein $\beta \gamma$-CAT have been proposed to facilitate the toad in the sense and uptake of environmental materials (like nutrients and antigens) and vesicular transport, while maintaining mucosal barrier function and fulfilling immune defense (17). Accordingly, the roles of $\beta \gamma$-CAT in immune defense have been first documented $(21,23-26)$.

Amphibian skin is a major organ responsible for water acquisition and maintaining $(1,3)$, and $\beta \gamma$-CAT is a major component of $B$. maxima skin secretions (18). In the present study, we found that the expression and localization of $\beta \gamma$-CAT in toad $B$. maxima are related to environmental osmotic conditions, and that this protein is able to counteract cellular dehydration under extracellular hyperosmosis. $\beta \gamma$-CAT stimulated and participated in cell macropinocytosis and exosome release, promoting water and $\mathrm{Na}^{+}$uptake and regulating AQP localization. Collectively, these results revealed that a secretory PFP can drive water acquisition and maintaining. 


\section{Results}

\section{$\beta \gamma$-CAT is involved in responses to osmotic stress.}

Amphibian skin has played a principal role in water balance during evolutionary adaptation to land environments $(1,2)$. Accordingly, when exposed to hypertonic Ringer's solution, toads (B. maxima) rapidly lost weight, which was gradually recovered when the animal was then placed into isotonic Ringer's solution (Fig. 1A). This suggests that the toad transports water through its skin under conditions of osmotic stress. Skin secretions play pivotal roles in the physiological functions of amphibian skin (2). Furthermore, $\beta \gamma$-CAT is a major proteinaceous component of skin secretions in toad B. maxima (18). Two subunits of $\beta \gamma$-CAT together were estimated to account for about $50 \%$ of proteins in B. maxima skin secretions (Fig. S1A). We speculated that toad skin secretions, especially $\beta \gamma$-CAT, might play an active role in water balance. Osmotic stress experiments were conducted to test the possible functions of $\beta \gamma$-CAT-containing skin secretions in maintenance of water balance in the toad. Toads B. maxima lost $20 \%$ of their body weight and $50 \%$ of the animals died when they were placed in the hypertonic Ringer's solution for 24 hours (Fig. $1 B$ and Fig. S1B). Toad skin secretions were depleted by electro-stimulation 30 minutes before the animals were placed in different osmotic solutions. Although no toad deaths were recorded after electro-stimulation in the isotonic Ringer's solution, significant mortality occurred in the hypertonic Ringer's solution (Fig. 1B). Therefore, it appears that skin secretions are critical for the toad to cope with hypertonic environments. To further investigate the possible involvement of $\beta \gamma$-CAT in the toad's water balance, real-time fluorescent quantitative PCR was used to detect the expression of two $\beta \gamma$-CAT subunits in the skin, UB and kidney of toad B. maxima during dehydration and weight recovery (water absorption after dehydration). It was found that $\beta \gamma$-CAT $\alpha$-subunit expression was upregulated in the skin and kidney during weight recovery via water uptake after dehydration (Fig. $1 C$ and Fig. S1C). In contrast, the expression of $\beta \gamma$-CAT $\beta$-subunit mRNA was upregulated in the toad skin 
during dehydration or water absorption (Fig. 1D), but not in the kidney (Fig. S1D). Interestingly, in the UB, the mRNA levels of the both subunits were increased when toads were exposed to hypertonic Ringer's solution, and returned to the normal level when toads were subsequently placed in isotonic Ringer's solution (Fig. $1 E$ and $F$ ). Therefore, $\beta \gamma$-CAT expression is associated with changes to external osmotic conditions.

In addition, we analyzed the expression and localization of $\beta \gamma$-CAT protein under the same treatment conditions via immunohistofluorescence (IHF). $\beta \gamma$-CAT was mainly concentrated within the epidermis and glands of the skin, and it was obviously upregulated in the epidermis and basement membrane during osmotic stress (Fig. 1G). $\beta \gamma$-CAT was predominantly localized within the transitional epithelium in the UB. The expression of $\beta \gamma$-CAT protein in toad UB was upregulated in hypertonic Ringer's solution and downregulated when the toad was then placed into isotonic Ringer's solution (Fig. 1H). Collectively, these results revealed an involvement of $\beta \gamma$-CAT in the toad's responses to osmotic stress.

\section{$\beta \gamma$-CAT counteracts cell dehydration under extracellular hyperosmosis.}

$\beta \gamma$-CAT is a vital functional protein of $B$. maxima, and its constitutive expression can be detected in various toad tissues $(18,23)$, including skin secretions and epithelial cells from the skin and UB, as well as kidney and peritoneal cells. Endogenous secretion of $\beta \gamma$-CAT in these toad-derived cells was further analyzed by a hemolysis assay, a sensitive method to detect the presence of biologically active $\beta \gamma$-CAT $(18,19)$. All the media of these cultured toad cells showed potent hemolytic activity, which was totally inhibited by anti- $\beta \gamma$-CAT antibodies, confirming the presence of secreted $\beta \gamma$-CAT (Fig. S2A). In addition, we measured the cytotoxicity of $\beta \gamma$-CAT to B. maxima cells. Previously, it was reported that $\beta \gamma$-CAT showed no cytotoxicity to toad peritoneal cells at dosages up to $400 \mathrm{nM}$ (23). The present study further determined that cytotoxicity of $\beta \gamma$-CAT to skin and UB epithelial cells and kidney cells of B. maxima occurred only when its concentration reached $2 \mu \mathrm{M}$ (Fig. $\mathrm{S} 2 B$ ), a level much higher than physiological concentrations $(20-100 \mathrm{nM}$, as 
determined in the toad peritoneum) (23). In contrast, mammalian cells are much more sensitive to $\beta \gamma$-CAT. Although $10 \mathrm{nM}$ of $\beta \gamma$-CAT showed no cytotoxicity to MDCK, Caco-2 and T24 cells, the protein caused significant cell death when dosages used were higher than 50-100 nM (Fig. S2C). Thus, in all subsequent experiments concerning the addition of purified $\beta \gamma$-CAT to cells, the protein dosages used were: epithelial cells from toad skin, $100 \mathrm{nM}$; UB epithelial cells, $50 \mathrm{nM}$; peritoneal cells, $50 \mathrm{nM}$; mammalian MDCK, $10 \mathrm{nM}$; Caco-2, $10 \mathrm{nM}$; and T24, $5 \mathrm{nM}$.

To further explore the potential role of $\beta \gamma$-CAT in water transport, we investigated whether the protein is able to counteract cell dehydration under extracellular hyperosmosis. We confirmed the action of $\beta \gamma$-CAT on toad UB epithelial cells, mammalian MDCK, Caco- 2 and T2 4 cells, as determined by the formation of $\beta \gamma$-CAT oligomers after treatment with the protein (Fig. $2 A$ ). $\beta \gamma$-CAT oligomers were detected in toad UB epithelial cells without the addition of the protein, possibly caused by endogenously secreted $\beta \gamma$-CAT (Fig. $2 A$ ). The addition of purified $\beta \gamma$-CAT further increased the concentration of oligomers of the protein in the UB epithelial cells (Fig. $2 A)$. Then, we analyzed the electrophysiological characteristics of $\beta \gamma$-CAT oligomers on outside-out patches of HEK293 cells. Currents were elicited by $500 \mathrm{~ms}$ ramp protocol between $-100 \mathrm{mV}$ to $+100 \mathrm{mV}$ every $2 \mathrm{~s}$ from a holding potential of $0 \mathrm{mV}$. $\beta \gamma$-CAT induced macroscopic currents were recorded and normalized, which has the characteristic of inward rectification (Fig. $2 B$ ). These proved that $\beta \gamma$-CAT could form transmembrane pores after oligomerization on the membrane and mediate ion flow. We further investigated ion selectivity of $\beta \gamma$-CAT channels through ion replacement experiments. Asymmetric 150:15 $\mathrm{mM} \mathrm{NaCl}$ solutions left-shifted the reversal potential from $0 \mathrm{mV}$ to $-61.2 \mathrm{mV}$, which is very close to the theoretical equilibrium potential of $\mathrm{Na}^{+}$, indicating that the $\beta \gamma$-CAT channels are permeable to $\mathrm{Na}^{+}$but not to $\mathrm{Cl}^{-}$. (Fig. $2 C$ ). These results demonstrated that the $\beta \gamma$-CAT channels allowed $\mathrm{Na}^{+}$ flow.

On this basis, we explored the potential role of $\beta \gamma$-CAT when cells were challenged by extracellular hyperosmosis using hypertonic solution. We observed that $\beta \gamma$-CAT promoted volume recovery of MDCK, Caco-2 and T24 cells in hypertonic solution 
(Fig. 2D), revealing the capacity of the protein to facilitate water uptake under hyperosmotic conditions. In consistency with those observations, immune-depletion of endogenous $\beta \gamma$-CAT further decreased the cell diameters of toad UB epithelial cells in hypertonic Ringer's solution relative to those in the absence of anti- $\beta \gamma$-CAT antibodies (Fig. 2E). It is well documented that AQPs can mediate the rapid cellular flow of water (27). Thus, it is necessary to clarify whether the cell volume recovery by water uptake mediated by $\beta \gamma$-CAT under osmotic stress was associated with AQP function. Sequence alignment and evolutionary analysis of B. maxima AQPs based on a toad UB transcriptome revealed that AQP1, AQP2, AQP3, AQP5 and AQP11 were present in B. maxima UB tissue (Fig. S2D). Because the mercury-sensitive sites of $B$. maxima AQPs (BmAQPs) were evolutionarily conserved (Fig. $\mathrm{S} 2 E$ ), $\mathrm{HgCl}_{2}$ was used to inhibit BmAQPs function. Cell volume was unchanged in hypertonic Ringer's solution after blocking BmAQPs with $\mathrm{HgCl}_{2}$. Conversely, cell volume recovery of toad UB epithelial cells via water uptake was clearly reduced after immunodepletion of endogenous $\beta \gamma$-CAT (Fig. $2 F$ ). Taken together, these results revealed the capacity of $\beta \gamma$-CAT to promote cell volume recovery by stimulating water acquisition during extracellular hyperosmosis, and indicated that the effect was independent of water flow via plasma membrane AQPs.

\section{B $\gamma$-CAT promotes macropinocytosis.}

$\beta \gamma$-CAT participates in cell volume regulation, suggesting its involvement in water transport. We next analyzed the possible cellular mechanism behind this interesting phenomenon. Water acquisition can be rapidly realized through AQPs and/or macropinocytosis $(12,27,28)$. Previously, in a murine dendritic cell (DC) model, preliminary data suggested that $\beta \gamma$-CAT might enhance pinocytosis (25). In the present study, we carefully studied the ability of this protein to stimulate and participate in macropinocytosis in various types of $B$. maxima cells. First, immunoelectron microscopy (IEM) revealed that $\beta \gamma$-CAT was localized in cellular pseudopodia and macropinosomes with diameters up to $300 \mathrm{~nm}$ formed by macropinocytosis in toad skin and UB tissues (Fig. 3A). $\beta \gamma$-CAT was also present in 
the intercellular spaces of epithelial cells (Fig. 3A). Lucifer Yellow (LY) and 70-kDa dextran are fluid-phase specific markers for macropinocytosis $(29,30)$. In epithelial cells obtained from toad skin and UB, and in toad kidney or peritoneal cells, immunodepletion of endogenous $\beta \gamma$-CAT by anti- $\beta \gamma$-CAT antibodies greatly decreased internalization of LY and FITC-dextran, relative to cells observed in the presence of control rabbit IgG (Fig. 3B, 3C, S3A and S3B). Furthermore, addition of $\beta \gamma$-CAT to cells from toad skin and UB, and mammalian MDCK and T24 cells substantially enhanced the internalization of LY and FITC-dextran (Fig. 3D, 3E, S3C and $\mathrm{S} 3 D$ ). Confocal microscopy showed that $\beta \gamma$-CAT was endocytosed and located in the macropinosomes of toad UB epithelial cells (Fig. S3E), peritoneal cells (Fig. S3F), and mammalian MDCK cells (Fig. S3G). In addition, we found that total $\mathrm{Na}^{+}$ concentrations in toad UB epithelial cells and MDCK treatment with $\beta \gamma$-CAT were 3.5 and 1.1 times of that of the control, respectively (Fig. $3 F$ ).

Next, the molecular mechanism of macropinocytosis induced by $\beta \gamma$-CAT was investigated using pharmacological agents. 5-(N-ethyl-N-isopropyl) amiloride (EIPA, a $\mathrm{Na}^{+} / \mathrm{H}^{+}$-exchanger inhibitor) and wortmannin (WORT, a PI3K inhibitor) are commonly used to inhibit macropinocytosis $(31,32)$. We found that EIPA and WORT reduced macropinocytosis induced by $\beta \gamma$-CAT both in toad UB epithelial cells and mammalian MDCK cells (Fig. $3 G$ and $H$ ). Moreover, $\beta \gamma$-CAT stimulated an increase in Akt phosphorylation in MDCK cells (Fig. 3I). Taken together, these results demonstrated that $\beta \gamma$-CAT, an endogenously secreted complex of a PFP and a TFF, stimulates and participates in macropinocytosis in diverse cells of B. maxima as well as in various mammalian cells.

\section{$\beta \gamma$-CAT in AQP regulation.}

AQPs and ion flux are involved in water transport and volume regulation of toad UB cells under stimulation by multiple hormones (33-35). Internalization by macropinocytosis is unselective, whereas transitional epithelial cells are polar. Therefore, we assessed the relationship between $\beta \gamma$-CAT-stimulated macropinocytosis and BmAQP2. We observed that the location of $\beta \gamma$-CAT and BmAQP2 in toad UB 
epithelial cells differed between isotonic, hypertonic and 'hypertonic/isotonic' (hypertonic followed by return to isotonic) Ringer's solutions (Fig. 4A). When toads were exposed to isotonic Ringer's solution, $\beta \gamma$-CAT and BmAQP2 were located together and were distributed on both the apical and basal sides of the transitional epithelium in toad UB. In hypertonic Ringer's solution, $\beta \gamma$-CAT and BmAQP2 shifted their location to the basal or lateral sides of the UB transitional epithelium. In contrast, when the toads were returned to isotonic Ringer's solution from hypertonic Ringer's solution, $\beta \gamma$-CAT and BmAQP2 began to migrate to the apical side of the transitional epithelium. This observation suggests that macropinocytosis induced by $\beta \gamma$-CAT is involved in the internalization and transport of BmAQP2 in the UB. In MDCK, intracellular colocalization of $\beta \gamma$-CAT and AQP2 or AQP3 was also observed (Fig. 4 $B$ and $C$ ). These results indicate that macropinocytosis stimulated by $\beta \gamma$-CAT plays a role in the regulation of AQPs under various osmotic conditions, revealing another aspect of this protein in the modulation of toad water balance.

\section{$\beta \gamma$-CAT enhances exosome release.}

The secretion of exosomes is an important aspect of cell exocytosis that can be viewed as a means of selective transport of materials among cells and a mode of intercellular communications (36). Because $\beta \gamma$-CAT promotes macropinocytosis, we further explored the cellular fate and role of $\beta \gamma$-CAT-containing vesicles. $\beta \gamma$-CAT was readily detected by IEM in both the multivesicular bodies (MVBs) and the intraluminal vesicles (ILVs) of B. maxima UB epithelial cells (Fig. 5A). Furthermore, it was noted that the survival rate of toad UB epithelial cells was greater than $98 \%$ after in vitro culture for 3 hours at room temperature (Fig. S4A). Thus, exosomes secreted by these cells were collected and their shapes were observed by TEM (Fig. $5 B)$. The exosome markers CD63, TSG101 and flotillion-1 were detected in the collected exosomes and were substantially augmented in UB epithelial cells treated by the addition of $50 \mathrm{nM}$ purified $\beta \gamma$-CAT (Fig. 5C). Nanoparticle tracking analysis (NTA) revealed that immunodepletion of endogenous $\beta \gamma$-CAT greatly attenuated exosome release from toad UB epithelial cells and peritoneal cells (Fig. 5D and Fig. 
$4 B$ ), while the addition of $\beta \gamma$-CAT substantially augmented exosome release from these cells (Fig. $5 E$ and Fig. S4C). It is noteworthy that none of these treatments changed the average exosome diameter. Similar results were also obtained in mammalian MDCK and T24 cells (Fig S4 D and E). Collectively, these results showed that $\beta \gamma$-CAT promotes the production and release of exosomes in both toad and mammalian cells.

We further investigated the properties of exosomes stimulated by $\beta \gamma$-CAT. The size distribution of exosomes derived from toad UB epithelial cells was determined by flow cytometry for nanoparticle analysis. The diameters of these exosomes were mainly concentrated in the range of $50-150 \mathrm{~nm}$ (Fig. S4F). When $1 \mathrm{mg} / \mathrm{mL}$ of FITC-dextran was incubated with isolated exosomes at room temperature for 3 hours, the exosomes did not contain FITC-dextran (Fig. S4G). In contrast, when dextran was added to the culture of toad UB epithelial cells under the same conditions, FITC-dextran was identified in exosomes secreted by the cells (Fig. S4G). This observation indicated that isolated exosomes did not take up $70-\mathrm{kDa}$ dextran, but dextran was taken up by toad UB epithelial cells by macropinocytosis and released from the cells in the form of exosomes. Interestingly, the proportion of exosomes containing FITC-dextran remained unchanged in toad UB epithelial cells with or without the addition of purified $\beta \gamma$-CAT (Fig. $5 F$ ), and there was no difference in the mean fluorescence intensity of these exosomes (Fig. 5G). These results suggested that $\beta \gamma$-CAT promotes exocytosis by increasing exosome release, facilitating the transcellular transport of extracellular substances like dextran. However, the apparent properties of exosomes released seem not altered as assayed at the present stage.

Both BmAQP2 and $\beta \gamma$-CAT oligomers were detected by western blotting in exosomes released from toad UB epithelial cells, reflecting the presence of endogenous $\beta \gamma$-CAT. The addition of $\beta \gamma$-CAT to the cells greatly enhanced the quantity of BmAQP2 and $\beta \gamma$-CAT oligomers detected (Fig. 5H). IEM demonstrated colocalization of BmAQP2 and $\beta \gamma$-CAT in individual exosomes (Fig. 5I). These observations suggest that $\beta \gamma$-CAT drives the extracellular recycling and/or intercellular communication of AQPs via exosome release. 
Furthermore, we analyzed the effect of $\beta \gamma$-CAT on $\mathrm{Na}^{+}$levels in exosomes from MDCK cells. No difference was observed for total $\mathrm{Na}^{+}$concentrations in exosomes produced by the same number of cells treated with or without $10 \mathrm{nM} \beta \gamma$-CAT (Fig. 5J). However, $\beta \gamma$-CAT increased exosome release of MDCK by 6.8 times (Fig. S4D), so the mean $\mathrm{Na}^{+}$concentrations of exosomes were significantly reduced (Fig. $5 K$ ). In addition, an increase in total $\mathrm{Na}^{+}$concentrations in exosomes induced by $\beta \gamma$-CAT were found in hypertonic media under the same conditions (Fig. $5 L$ ). 


\section{Discussion}

Amphibians live both on land and in water, and their skin is naked and acts as an important organ in water balance, respiration (gas exchange) and immune defense (1, 3). Though initially identified in B. maxima skin secretions (19), the PFP complex $\beta \gamma$-CAT is widely expressed in B. maxima water balance organs, including skin, UB and kidney (Fig $1 C$ - $H$, Fig. S1 $C$ and $D$ ). Previous studies using RT-PCR likewise indicated the expression of $\beta \gamma$-CAT in various $B$. maxima tissues $(23,24)$. Interestingly, in contrast to its potent cytotoxicity to various mammalian cells $(19,25)$, $\beta \gamma$-CAT only shows toxic effects in toad cells in a dosage up to $2 \mu \mathrm{M}$ (Fig. S2B), which is much higher than $\beta \gamma$-CAT physiological concentrations observed (23). Its toxicity to mammalian cells could reflect the absence of regulatory mechanisms for this exogenous PFP protein, which are normally present in the toad $(18,22)$. These observations support the notion that the PFP complex $\beta \gamma$-CAT can not be simply viewed as a cell death inducer and/or a microbicide, and besides its roles in immune defense $(17,23,24)$, the protein exhibits other essential physiological functions in this toad.

B. maxima skin is covered with skin secretions containing various biological molecules that fulfill a range of physiological function, including hormone-like peptides, antimicrobial peptides, af-PFPs, TFFs, and haem b-containing albumin (16, $37,38)$. Indeed, our results revealed that the skin secretions are necessary to prevent dehydration (Fig $1 B$ ). We further demonstrated that a major skin secretion component $\beta \gamma$-CAT has a role in toad water homeostasis. This PFP complex possesses the capacity to stimulate and participate in macropinocytosis (endocytosis) and exosome release (exocytosis) by epithelial cells of toad osmoregulatory organs, which promotes water and $\mathrm{Na}^{+}$transport involved in water homeostasis.

Previous studies with mammalian cells and toad peritoneal cells showed that $\beta \gamma$-CAT exerts its biological actions by endocytosis and the formation of channels on endolysosomes $(17,19,38)$, but the endocytic pathway of the protein remains elusive. Macropinocytosis, also referred to as cell drinking, is a form of endocytosis that 
mediates the non-selective uptake of extracellular fluid and solutes $(6,12)$. In a murine DC model, preliminary data showed that $\beta \gamma$-CAT increases the internalization of ovalbumin (antigen), presumed to be mediated by enhanced macropinocytosis (25). The present study carried out on various toad-derived cells, including epithelial cells from osmoregulatory organs and peritoneal cells, clearly showed that $\beta \gamma$-CAT is capable of inducing and participating in macropinocytosis. Macropinocytosis stimulated by $\beta \gamma$-CAT has been shown to facilitate the entry of water and $\mathrm{Na}^{+}$into cells as assayed in epithelial cells from toad osmoregulatory organs (Fig. $2 F$ and $3 F$ ). This may explain the role of $\beta \gamma$-CAT in promoting the recovery of cell volume under hypertonic stress (Fig. $2 D-F$ ). Growth factor-induced macropinocytosis is mediated by the activation of the Ras and PI3-kinase signaling pathways (11). Similarly, activation of PI3-kinase and Akt signaling was involved in macropinocytosis stimulated by $\beta \gamma$-CAT, as suggested by the effects of pharmacological inhibitors and Akt phosphorylation analysis (Fig. $3 G-I$ ). However, in contrast to classic growth factors, which bind to membrane protein receptors to initiate their signaling, $\beta \gamma$-CAT targets gangliosides and sulfatides as receptors in lipid rafts to initiate its cellular effects (22). The signals downstream from these lipid components, and their relationship to the induction of macropinocytosis, are presently unclear and are worthy of further study. In this respect, possible differences between macropinocytosis stimulated by growth factors and that induced by $\beta \gamma$-CAT should be investigated.

While engulfing large volumes of fluid, macropinocytosis also internalizes cell surface proteins such as receptors and integrins $(39,40)$. It has been proposed that endocytosis stimulated by $\beta \gamma$-CAT plays a role in the sorting of specific plasma membrane elements, such as functional integrated proteins or lipid components, which could help regulate cell responses to environmental variations (17). In addition, internalization and recycling of AQPs between the plasma membrane and the endosomal compartment have roles in controlling water uptake and conservation (5, 27). Accordingly, colocalization of $\beta \gamma$-CAT with AQPs was observed in endocytic organelles (Fig. 4), suggesting macropinocytosis induced by this PFP protein drives 
endocytosis and recycling of AQPs. Clearly, the presence of AQPs in the plasma membrane might facilitate water loss when cells faced by hypertonic osmotic stress, thus internalization of AQPs induced by $\beta \gamma$-CAT could counteract dehydration by preventing rapid water efflux and cell shrinkage.

The secretion of extracellular vesicles comprising exosomes and microvesicles represents a novel mode of intercellular communication and material exchange (36, 41). Previously, $\beta \gamma$-CAT was found to augment $\beta \gamma$-CAT-containing exosome release from murine DCs, which activates T cell response effectively (25). Because $\beta \gamma$-CAT is a factor exogenous to murine DCs, the explanation of the phenomenon is not obvious. However, the PFP is an endogenous element to toad cells. The present study illustrated that cell exocytosis in the form of exosome release was indeed augmented in the presence of $\beta \gamma$-CAT, as determined in diverse toad-derived cells (Fig. $5 D, 5 E$, $\mathrm{S} 4 B$ and $\mathrm{S} 4 C$ ), revealing that mediation of cell exocytosis via exosome release is an intrinsic property of this PFP protein. Previous studies demonstrated that $\beta \gamma$-CAT characteristically neutralizes the acidification of endocytic organelles containing this protein (23-25). This cellular process may result in the transformation of $\beta \gamma$-CAT-containing endocytic organelles into MVBs that does not fuse with lysosomes for the degradation of contained solutes. The observation that extracellular dextran, a tracer for $\beta \gamma$-CAT-induced endocytosis, was present in exosomes containing $\beta \gamma$-CAT (Fig. $5 F$ and $G$ ) supports this view.

The observation that $\beta \gamma$-CAT both stimulates and participates in macropinocytosis and exosome release strongly implies that this PFP is concerned with transcellular transport of internalized extracellular substances by PFP-driven vesicular transportation. This property is particularly important in vivo, where $\beta \gamma$-CAT could transport external substances such as water and $\mathrm{Na}^{+}$to internal environments of toad tissues without disruption of the cellular tight junctions that maintain epithelial barrier functions. Interestingly, AQPs were identified in exosomes released in the presence of $\beta \gamma$-CAT (Fig $5 H$ and $I$ ), suggesting that $\beta \gamma$-CAT modulates water homeostasis by driving extracellular recycling of, and/or intercellular communication via these water channels. Alternatively, $\beta \gamma$-CAT-mediated exocytosis might function as a means for 
expulsion of noxious and indigestible solutes contained in fluid macropinocytosed by cells for water acquisition, like the expelling of dextran from toad UB epithelial cells (Fig. $5 F$ and $G$ ). The role of $\beta \gamma$-CAT in expelling noxious substances engulfed by macropinocytosis via exocytosis induction and the cellular sorting mechanism involved are important future challenges.

It is generally understood that classic membrane integrated proteins, like AQPs and ion channels play fundamental roles in water transport and homeostasis $(27,42)$. Furthermore, the tight junction protein claudin-2 mediates the permeability to transepithelial water movements under osmotic or $\mathrm{Na}^{+}$gradients through the paracellular pathway (43-45). The present study has introduced a new player, a secretory endolysosome channel protein $\beta \gamma$-CAT, into the network of water transport and homeostasis via modulation of cellular endocytic and exocytic pathways. A cellular working model of the PFP $\beta \gamma$-CAT in promoting water uptake and maintaining was proposed in toad B. maxima (Fig. 6). In this model, water and $\mathrm{Na}^{+}$ can be taken up by macropinocytosis induced by the PFP $\beta \gamma$-CAT. Meanwhile, the transmembrane channels formed by $\beta \gamma$-CAT can mediate the $\mathrm{Na}^{+}$flow (Fig $2 B$ and $C$ ). Previous studies have shown that the channel formed by $\beta \gamma$-CAT can cause cation ion flux (46). The PFP complex targets viral envelope to form pores that induced potassium and calcium ion efflux (21). Unexpectedly, the average $\mathrm{Na}^{+}$concentrations of $\beta \gamma$-CAT-containing exosomes were substantially lower than those of the control (Fig $5 J$ and $K$ ). This phenomenon suggested that the presence of channels formed by $\beta \gamma$-CAT can lead to rapid efflux of $\mathrm{Na}^{+}$out of endolysosomes and/or exosomes. Thus, $\mathrm{Na}^{+}$can be release into cytosol through $\beta \gamma$-CAT channels in endocytic organelles, which drives water release to the cytosol via AQPs (Fig. 4). This is similar to the situation of two-pore channels working in mammalian macrophages $(39,40)$. Enhanced exosome release mediated by $\beta \gamma$-CAT may facilitate vesicular transport of ions such as $\mathrm{Na}^{+}$(Fig. 5L), and/or water, into the intercellular spaces below tight junctions favoring water absorption into deeper cell environments. Meanwhile, membrane-integrated ion channels and AQPs in the basal plasma membrane of epithelial cells could mediate the efflux of $\mathrm{Na}^{+}$and water into internal tissues 
underneath these cells achieving the absorption/reabsorption of water into toad internal tissues $(5,47)$. Drinking water by macropinocytosis is energetically expensive (12). However, the function of secretory PFP $\beta \gamma$-CAT in water acquisition and maintaining is necessary in light of various osmotic conditions that toad B. maxima has to face throughout its life cycle.

Secretory PFPs have been identified in organisms from all kingdoms of life $(15,16)$, and af-PFPs are widely distributed in plants and animals (15-17). The present study provides functional evidence to support our previous hypothesis on the SELC (secretory endolysosome channel) pathway mediated via a secretory PFP in cellular endocytic and exocytic systems (17). Regulated relying on environmental cues (17, 18), SELC protein $\beta \gamma$-CAT is characterized of driving intracellular intake and transport of extracellular materials and/or plasma membrane components in a vesicle form. These capacities raise the possibility that besides working in water maintaining, $\beta \gamma$-CAT and/or its homologues could play active and fundamental roles in cell metabolism, as previously proposed (17). These SELC proteins and classic membrane integrated proteins (like solute carrier family members) can form two coordinated cell arms in extracellular nutrient sensing, sampling and acquiring. Furthermore, it is possible that $\beta \gamma$-CAT and its homologues are involved in modulating cellular metabolic responses to nutrient variation and shortage in toad B. maxima. Obviously, these SELC proteins should be essential to cells in those cases that classic membrane solute carriers are blockaded or even absent. Our findings will serve as clues for uncovering the physiological roles of PFPs in other living organisms. Specifically, proliferating embryonic cells of oviparous animals depend entirely on catabolism of extracellular macromolecules deposited in the egg white and yolk (10). If macropinocytosis-driven by a PFP plays a role in oviparous animal embryonic development is an interesting subject for further investigation.

In conclusion, the current work elucidated an unexpected role of a secretory PFP in water transport and homeostasis. $\beta \gamma$-CAT, an af-PFP and a TFF complex, induce and participate in macropinocytosis and exosome release in epithelial cells from $B$. maxima osmoregulatory organs. These effects could explain the role of the PFP 
complex in promoting the internalization, transport and release of water, $\mathrm{Na}^{+}$and AQPs, working together in the maintenance of toad cell volume regulation and water homeostasis (Fig. 6). Together with membrane-integrated AQPs and ion channels, the action of secretory $\beta \gamma$-CAT can promote the overall achievement of water maintaining and balance, especially when the toad encounters osmotic stress. 


\section{Materials and Methods}

\section{Animals}

Toads (B. maxima) were captured in the wild and raised at room temperature by feeding with live Tenebrio molitor. Toads with an average weight of $19 \pm 5 \mathrm{~g}$ were used in experiments after fasting in isotonic Ringer's solution for 3 days. All the procedures and the care and handling of the animals were approved by the Institutional Animal Care and Use Committee at Kunming Institute of Zoology, Chinese Academy of Sciences (Approval ID: IACUC-OE-2021-05-001).

\section{Animal experiment}

Formulation of isotonic Ringer's solution $(111.2 \mathrm{mM} \mathrm{NaCl}, 1.9 \mathrm{mM} \mathrm{KCl}, 1.1 \mathrm{mM}$ $\mathrm{CaCl}_{2}, 2.4 \mathrm{mM} \mathrm{NaHCO}$, $1.6 \mathrm{mM} \mathrm{MgCl}_{2}$ ) was modified based on a previous report (48). After fasting, toads in the hypertonic group were placed in hypertonic Ringer's solution (Ringer's solution with $222.4 \mathrm{mM} \mathrm{NaCl}$ ) for 3 hours and their weight changes were recorded. Toads in the hypertonic/isotonic group were then transferred from hypertonic Ringer's solution to isotonic Ringer's solution for 3 hours and their weight changes were recorded. Weight changes of toads in isotonic Ringer's solution were recorded for 6 hours as a reference, termed the isotonic group. The skin of some toads was electrically stimulated $(4.5 \mathrm{~V} \mathrm{DC}$, pulse duration $10 \mathrm{~ms})$ for 3 minutes to deplete skin secretions, and the animals were placed in isotonic Ringer's solution for 30 minutes. Their survival rates were then recorded after 48 hours in isotonic and hypertonic solutions.

\section{Cell culture}

Toad epithelial cells were obtained by tissue digestion. Specifically, the UB, skin, and kidney tissue were dissected from five toads whose spinal cord had been destroyed. Tissues were further rinsed and stripped in Ringer's solution to remove residual blood, mucus and other impurities. They were then cut into pieces and washed twice in Ringer's solution, followed by oscillatory digestion with trypsin at 
room temperature for 40 minutes. The cells were separated with a 200 -mesh sieve. In addition, toad peritoneal cells were extracted from the peritoneal fluid of B. maxima. All cells were centrifugally enriched with $2,000 \mathrm{rpm}$ for $5 \mathrm{~min}$ at $4^{\circ} \mathrm{C}$. The digestive cells in toad skin and UB tissues contain around $90 \%$ and $60 \%$ of epidermal cells, respectively, by flow cytometry using an anti-pan cytokeratin AE1/AE3 monoclonal antibody (Thermo Fisher Scientific, Rockford, IL, USA).

\section{Additional Assays}

All other materials and methods are described in Materials and Methods of Supplemental Information. 


\section{References}

1. P. J. Bentley, Adaptations of amphibia to arid environments. Science 152, 619-623 (1966).

2. V. Shoemaker, K. A. Nagy, Osmoregulation in amphibians and reptiles. Annu. Rev. Physiol. 39, 449-471 (1977).

3. S. D. Hillyard, Behavioral, molecular and integrative mechanisms of amphibian osmoregulation. J. Exp. Zool. 283, 662-674 (1999).

4. Y. Ogushi, et al., The water-absorption region of ventral skin of several semiterrestrial and aquatic anuran amphibians identified by aquaporins. Am. J. Physiol.-Regul. Integr. Comp. Physiol. 299, R1150-R1162 (2010).

5. M. Suzuki, T. Hasegawa, Y. Ogushi, S. Tanaka, Amphibian aquaporins and adaptation to terrestrial environments: A review. Comp. Biochem. Physiol. A. Mol. Integr. Physiol. 148, 72-81 (2007).

6. M. C. Kerr, R. D. Teasdale, Defining macropinocytosis. Traffic 10, 364-371 (2009).

7. J. A. Swanson, C. Watts, Macropinocytosis. Trends Cell Biol. 5, 424-428 (1995).

8. C. Ramirez, A. D. Hauser, E. A. Vucic, D. Bar-Sagi, Plasma membrane V-ATPase controls oncogenic RAS-induced macropinocytosis. Nature 576, 477-481 (2019).

9. M. Bretou, et al., Lysosome signaling controls the migration of dendritic cells. Sci. Immunol. 2 (2017).

10. W. Palm, C. B. Thompson, Nutrient acquisition strategies of mammalian cells. Nature 546, 234-242 (2017).

11. W. Palm, Metabolic functions of macropinocytosis. Philos. Trans. R. Soc. B Biol. Sci. 374, 20180285 (2019).

12. J. A. Swanson, J. S. King, The breadth of macropinocytosis research. Philos. Trans. R. Soc. B Biol. Sci. 374, 20180146 (2019).

13. M. W. Parker, et al., Structure of the Aeromonas toxin proaerolysin in its water-soluble and membrane-channel states. Nature 367, 292-295 (1994).

14. M. D. Peraro, F. G. van der Goot, Pore-forming toxins: ancient, but never really out of fashion. Nat. Rev. Microbiol. 14, 77-92 (2016).

15. P. Szczesny, et al., Extending the aerolysin family: from bacteria to vertebrates. PLoS ONE 6, e20349 (2011).

16. Y. Zhang, Why do we study animal toxins? Zool. Res. 36, 183-222 (2015). 
17. Y. Zhang, Q. Q. Wang, Z. Zhao, C. J. Deng, Animal secretory endolysosome channel discovery. Zool. Res. 42, 141-152 (2021).

18. Q. Q. Wang, et al., A cellular endolysosome-modulating pore-forming protein from a toad is negatively regulated by its paralog under oxidizing conditions. J. Biol. Chem. 295, 10293-10306 (2020).

19. S. B. Liu, et al., A novel non-lens $\beta \gamma$-crystallin and trefoil factor complex from amphibian skin and its functional implications. PLOS ONE 3, e1770 (2008).

20. Q. Gao, et al., Characterization of the $\beta \gamma$-crystallin domains of $\beta \gamma$-CAT, a non-lens $\beta \gamma$-crystallin and trefoil factor complex, from the skin of the toad Bombina maxima. Biochimie 93, 1865-1872 (2011).

21. L. Liu, et al., An aerolysin-like pore-forming protein complex targets viral envelope to inactivate Herpes Simplex Virus Type 1. J. Immunol. (2021).

22. X. L. Guo, et al., Endogenous pore-forming protein complex targets acidic glycosphingolipids in lipid rafts to initiate endolysosome regulation. Commun. Biol. 2, 59 (2019).

23. Y. Xiang, et al., Host-derived, pore-forming toxin-like protein and trefoil factor complex protects the host against microbial infection. Proc. Natl. Acad. Sci. 111, 6702-6707 (2014).

24. S. A. Li, et al., Host pore-forming protein complex neutralizes the acidification of endocytic organelles to counteract intracellular pathogens. J. Infect. Dis. 215, 1753-1763 (2017).

25. C. J. Deng, et al., A secreted pore-forming protein modulates cellular endolysosomes to augment antigen presentation. FASEB J. 34, 13609-13625 (2020).

26. Z. H. Gao, et al., Pore-forming toxin-like protein complex expressed by frog promotes tissue repair. FASEB J. 33, 782-795 (2019).

27. K. Takata, Aquaporins: water channel proteins of the cell membrane. Prog. Histochem. Cytochem. 39, 1-83 (2004).

28. A. de Baey, A. Lanzavecchia, The role of aquaporins in dendritic cell macropinocytosis. J. Exp. Med. 191, 743-748 (2000).

29. M. Nonnenmacher, T. Weber, Adeno-associated virus 2 infection requires endocytosis through the CLIC/GEEC pathway. Cell Host Microbe 10, 563-576 (2011).

30. J. P. Lim, P. A. Gleeson, Macropinocytosis: an endocytic pathway for internalising large gulps. Immunol. Cell Biol. 89, 836-843 (2011).

31. N. Araki, M. T. Johnson, J. A. Swanson, A role for phosphoinositide 3-kinase in the completion of macropinocytosis and phagocytosis by macrophages. J. Cell Biol. 135, 1249-1260 (1996). 
32. H. P. Lin, et al., Identification of novel macropinocytosis inhibitors using a rational screen of Food and Drug Administration-approved drugs. Br. J. Pharmacol. 175, 3640-3655 (2018).

33. R. M. Hays, A. Leaf, Studies on the movement of water through the isolated toad bladder and its modification by vasopressin. J. Gen. Physiol. 45, 905-919 (1962).

34. T. Hasegawa, M. Suzuki, S. Tanaka, Immunocytochemical studies on translocation of phosphorylated aquaporin-h2 protein in granular cells of the frog urinary bladder before and after stimulation with vasotocin. Cell Tissue Res. 322, 407-415 (2005).

35. C. W. Davis, A. L. Finn, Interactions of sodium transport, cell volume, and calcium in frog urinary bladder. J. Gen. Physiol. 89, 687-702 (1987).

36. G. van Niel, G. D'Angelo, G. Raposo, Shedding light on the cell biology of extracellular vesicles. Nat. Rev. Mol. Cell Biol. 19, 213-228 (2018).

37. Y. X. Zhang, R. Lai, W. H. Lee, Y. Zhang, Frog albumin is expressed in skin and characterized as a novel potent trypsin inhibitor. Protein Sci. 14, 2469-2477 (2005).

38. F. Zhao, et al., Comprehensive transcriptome profiling and functional analysis of the frog (Bombina maxima) immune system. DNA Res. 21, 1-13 (2014).

39. S. A. Freeman, et al., Lipid-gated monovalent ion fluxes regulate endocytic traffic and support immune surveillance. Science 367, 301-305 (2020).

40. J. S. King, E. Smythe, Water loss regulates cell and vesicle volume. Science 367, 246-247 (2020).

41. A. E. Russell, et al., Biological membranes in EV biogenesis, stability, uptake, and cargo transfer: an ISEV position paper arising from the ISEV membranes and EVs workshop. J. Extracell. Vesicles 8, 1684862 (2019).

42. M. L. McManus, K. B. Churchwell, K. Strange, Regulation of cell volume in health and disease. N. Engl. J. Med. 333, 1260-1267 (1995).

43. R. Rosenthal, et al., Claudin-2, a component of the tight junction, forms a paracellular water channel. J. Cell Sci. 123, 1913-1921 (2010).

44. A. Wilmes, L. Aschauer, A. Limonciel, W. Pfaller, P. Jennings, Evidence for a role of claudin 2 as a proximal tubular stress responsive paracellular water channel. Toxicol. Appl. Pharmacol. 279, 163-172 (2014).

45. R. Rosenthal, et al., Claudin-2-mediated cation and water transport share a common pore. Acta Physiol. 219, 521-536 (2017).

46. Q. Gao, et al., $\beta Y$-CAT, a non-lens betagamma-crystallin and trefoil factor complex, induces calcium-dependent platelet apoptosis. Thromb. Haemost. 105, 846-854 (2011). 
bioRxiv preprint doi: https://doi.org/10.1101/2021.07.31.454564; this version posted July 31, 2021. The copyright holder for this preprint

(which was not certified by peer review) is the author/funder, who has granted bioRxiv a license to display the preprint in perpetuity. It is made available under aCC-BY 4.0 International license.

47. Y. Marunaka, Characteristics and pharmacological regulation of epithelial $\mathrm{Na}+$ channel (ENaC) and epithelial Na+ transport. J. Pharmacol. Sci. 126, 21-36 (2014).

48. A. Edström., Effects of $\mathrm{Ca} 2+$ and $\mathrm{Mg} 2+$ on rapid axonal transport of proteins in vitro in frog sciatic nerves. J. Cell Biol. 61, 812-818 (1974). 


\section{Acknowledgements}

This work was supported by grants from the National Natural Science Foundation of China (grant numbers 31872226, U1602225 and 31572268) and the Yunling Scholar Program to Yun Zhang. We would like to thank the Kunming Biological Diversity Regional Center of Instrument, Kunming Institute of Zoology, Chinese Academy of Sciences for our electron microscopy and we would be grateful to Yingqi Guo for her help of making EM samples.

\section{Author contributions}

Y.Z., Z.Z., and Z.H.S conceived of and conceptualized the study; Z.Z., Z.H.S., and C.J.Y did the experiments, Z.Z., Y.Z., Z.H.S, and C.J.Y analyzed and interpreted the data; Z.Z., Y.Z., Z.H.S wrote the manuscript; Y.Z., Z.Z., Z.H.S and C.J.Y critically revised the manuscript for important intellectual content.

\section{Competing Interests statement}

We declare that we have no conflicts of interest. 
bioRxiv preprint doi: https://doi.org/10.1101/2021.07.31.454564; this version posted July 31, 2021. The copyright holder for this preprin (which was not certified by peer review) is the author/funder, who has granted bioRxiv a license to display the preprint in perpetuity. It is made available under aCC-BY 4.0 International license.

A

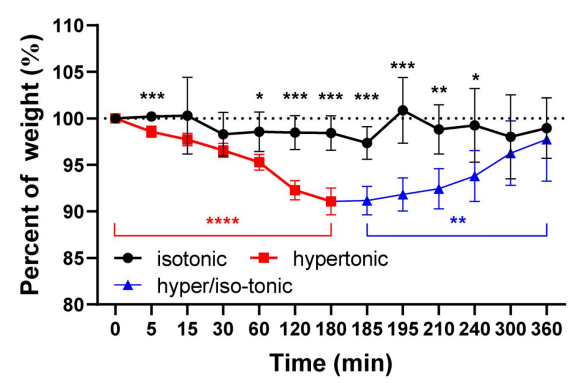

C

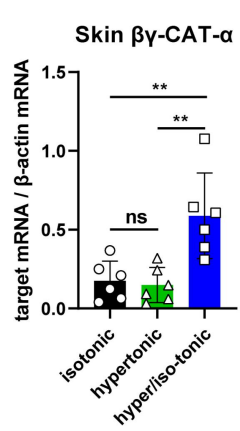

G

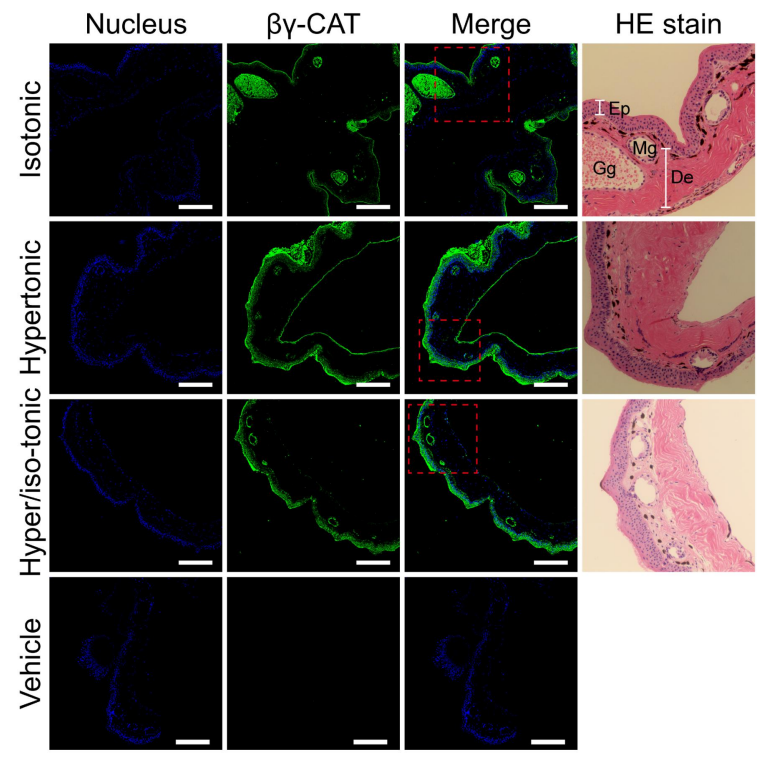

B

$\mathrm{E}$
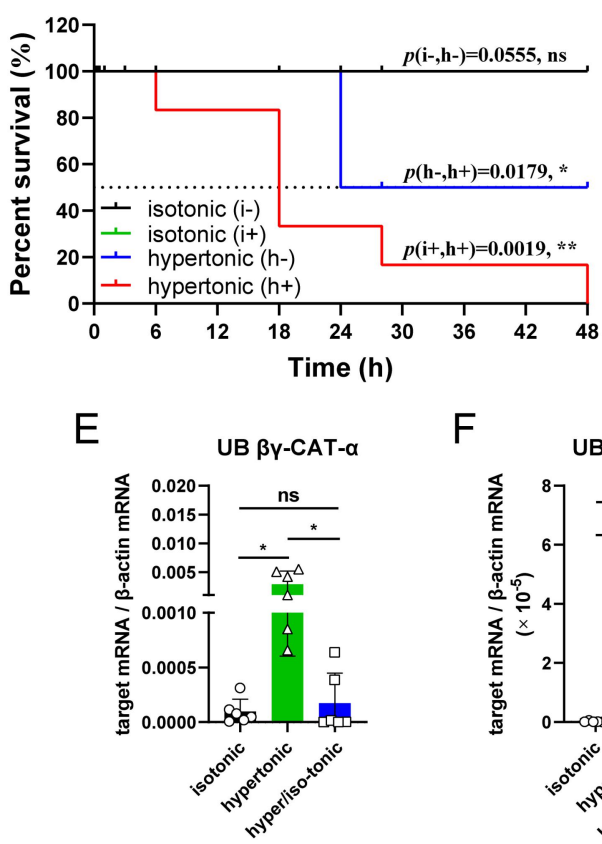

$\mathrm{F}$ UB $\beta Y-C A T-\beta$

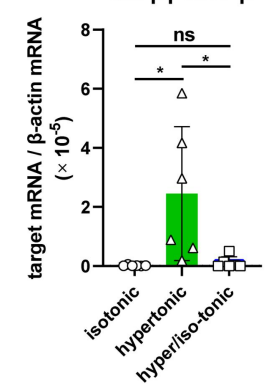

$\mathrm{H}$

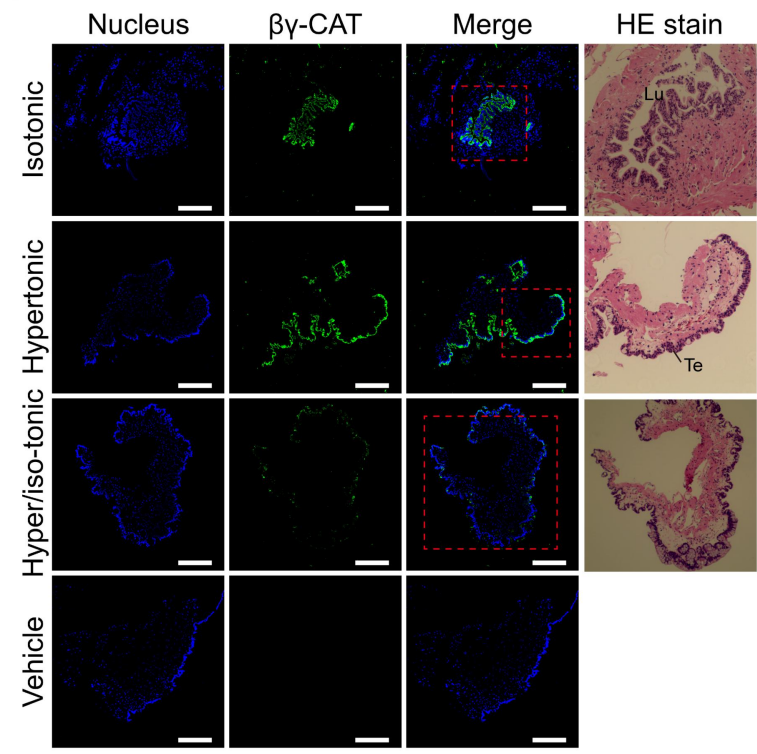

Fig. 1. $\boldsymbol{\beta} \gamma$-CAT is involved in responses to osmotic stress. $(A)$ Toad weight changes

were measured after placing them in isotonic, hypertonic and hypertonic/isotonic Ringer's solutions. Initial weight of toads is $19 \pm 5 \mathrm{~g}(n=5)$. (B) Survival rates of toads in isotonic (i) and hypertonic (h) Ringer's solution were determined after 48 hours. The animals were placed in each of the solutions after 30 min with $(+)$ or without (-) electro-stimulation to delete toad skin secretions $(n=6) .(C-F)$ The expression of $\beta \gamma$-CAT subunits in toad skin and UB were analyzed by real-time fluorescent quantitative PCR $(n=6)$. The animals were placed in isotonic or 
hypertonic Ringer's solution for 3 hours before the samples were collected. In the hypertonic/isotonic group, the toad was first placed in the hypertonic solution for 3 hours, moved to isotonic solution for a further 3 hours, and then the sample was collected. $(G, H)$ Following placement of toads in isotonic, hypertonic or hypertonic/isotonic Ringer's solution as described above, the localization and expression of $\beta \gamma$-CAT in the toad skin $(G)$ and UB $(H)$ tissues were analyzed by immunohistofluorescence (IHF). Ep, epidermis; De, dermis; Mg, mucous gland; Gg, granular gland; Lu, lumen; Te, transitional epithelium, Scale bars, $500 \mu \mathrm{m}$. All data represent the mean $\pm \mathrm{SD}$ of two independent replicated experiments. $* P<0.05$ and ${ }^{* *} P<0.01$ by the Gehan-Breslow-Wilcoxon test in survival rate analysis. $\mathrm{ns}(P \geq$ $0.05) ; * P<0.05, * * P<0.01$ and $* * * P<0.001$ by unpaired $t$ test in other experiments. All data are representative of at least two independent experiments in $G, H$. See also Fig. S1. 

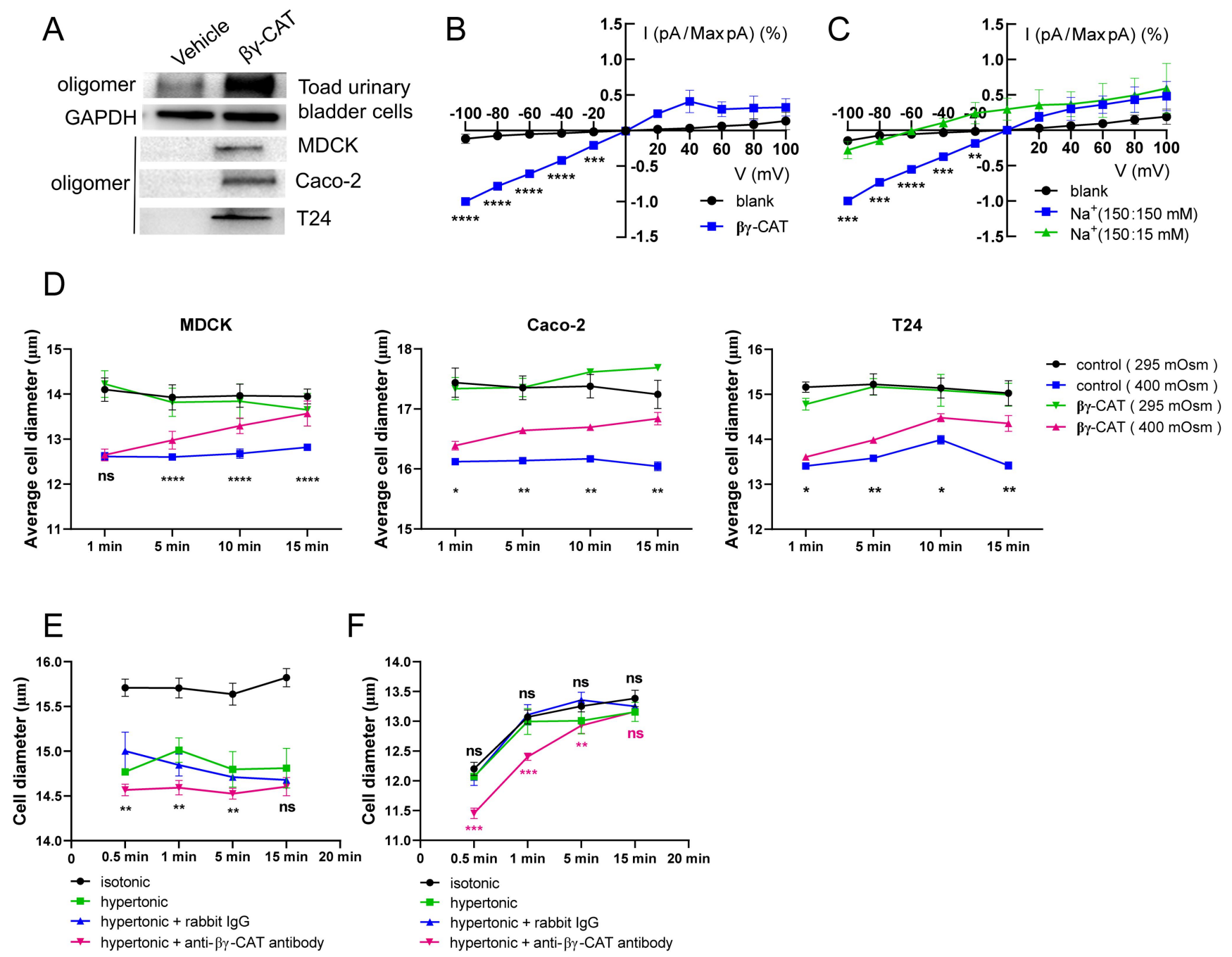

Fig. 2. $\beta \gamma$-CAT counteracts cellular dehydration under extracellular

hyperosmosis. $(A)$ Various mammalian and toad cells were first treated with or without $\beta \gamma$-CAT for 15 minutes. The appearance of $\beta \gamma$-CAT oligomers in the treated cells was determined by western blotting. (B) Normalized current-voltage curves of channels formed by $100 \mathrm{nM} \beta \gamma$-CAT on HEK293 cells. Currents were elicited by 500 ms ramp protocol between $-100 \mathrm{mV}$ to $+100 \mathrm{mV}$ every $2 \mathrm{~s}$ from a holding potential of $0 \mathrm{mV}(n=3)$. (C) Normalized current-voltage curves of $\beta \gamma$-CAT channels in indicated solutions (pipette/bath) $(n=3) . \mathrm{Na}^{+}(150: 15 \mathrm{mM})$ represents the $150 \mathrm{mM}$ $\mathrm{Na}^{+}$in the pipette and $15 \mathrm{mM} \mathrm{Na}^{+}$in the bath. $(D)$ Diameter changes of MDCK, Caco-2 and T24 cells in isotonic (295 mOsm) or hypertonic (400 mOsm) PBS in the presence or absence of $\beta \gamma$-CAT as determined by using a cell counter. Digested cells were first suspended in PBS for 15 minutes before they were used in this experiment. $(E, F)$ Diameter changes of toad UB epithelial cells in isotonic (black) and hypertonic 
(green) Ringer's solution in the presence of $50 \mu \mathrm{g} / \mathrm{mL}$ rabbit antibody (blue) or anti- $\beta \gamma$-CAT antibody (magenta). In $(F)$, the cells were first treated with $0.3 \mathrm{mM}$ $\mathrm{HgCl}_{2}$ for 10 minutes before cell diameter measurement with a cell counter. In all experiments shown in Figure 2, $\beta \gamma$-CAT dosages used were $10 \mathrm{nM}$ for MDCK and Caco-2, $5 \mathrm{nM}$ for T24 and $50 \mathrm{nM}$ for toad UB epithelial cells. The bars represent the mean $\pm \mathrm{SD}$ of triplicate samples in $B-E$. The bars represent the mean $\pm \mathrm{SD}$ of three independent replicates in $F$. ns $(P \geq 0.05),{ }^{*} P<0.05, * * P<0.01, * * * P<0.001$ and $* * * * P<0.0001$ by the unpaired $t$ test. All data are representative of at least two independent experiments. See also Fig. S2. 
A

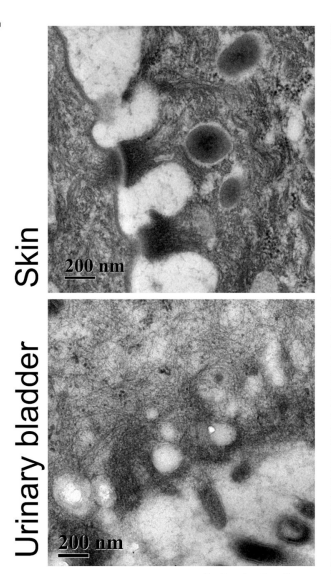

Vehicle

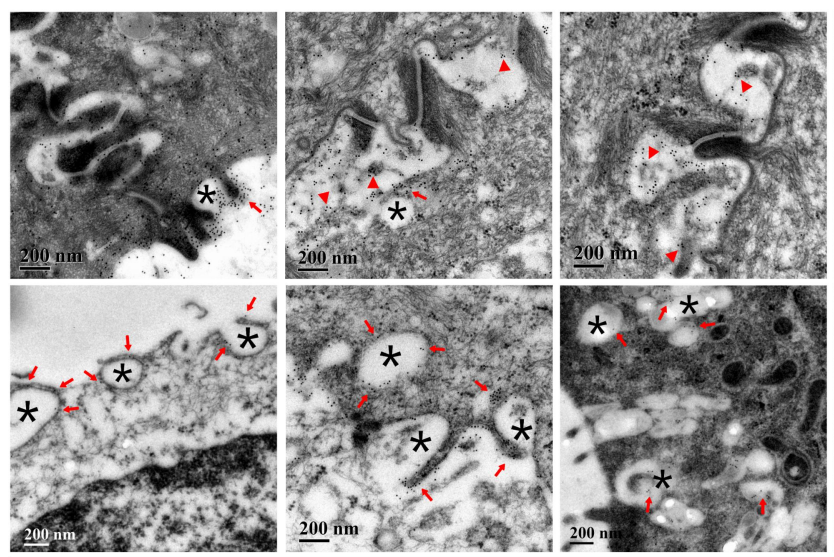

B

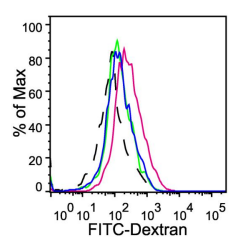

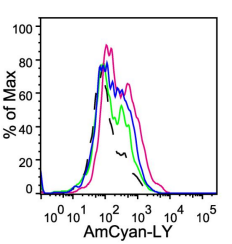
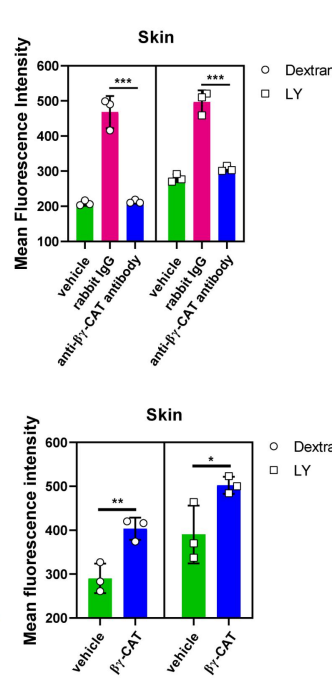

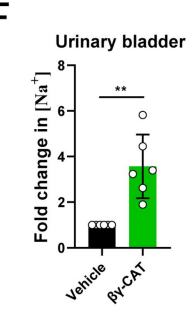

D
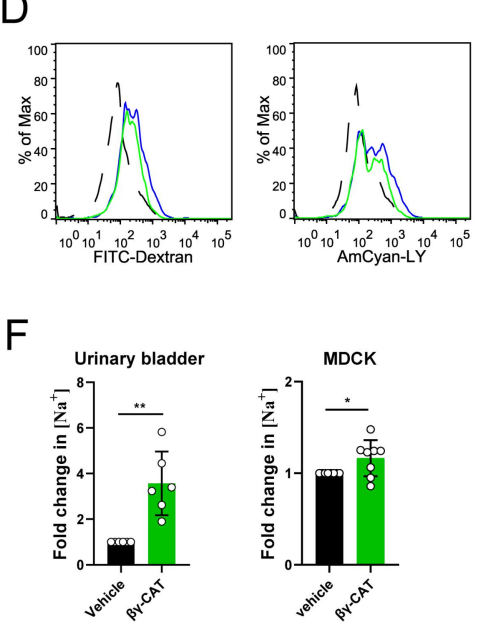

C
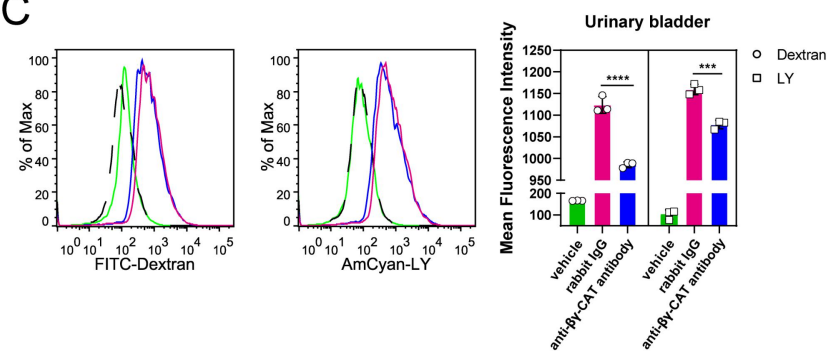

E
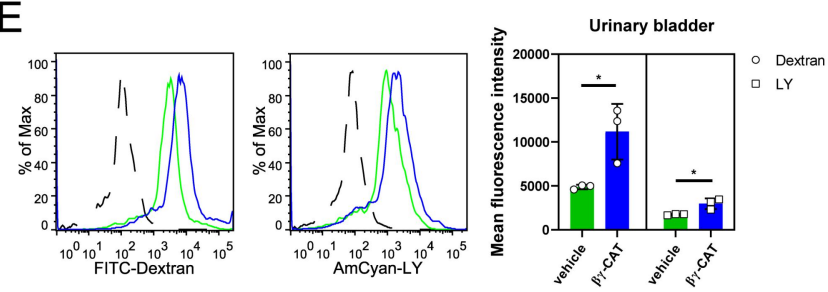

G

$\mathrm{H}$
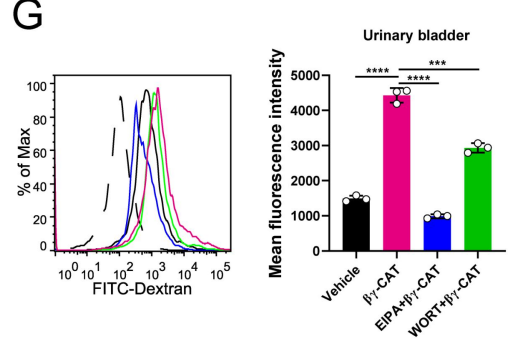

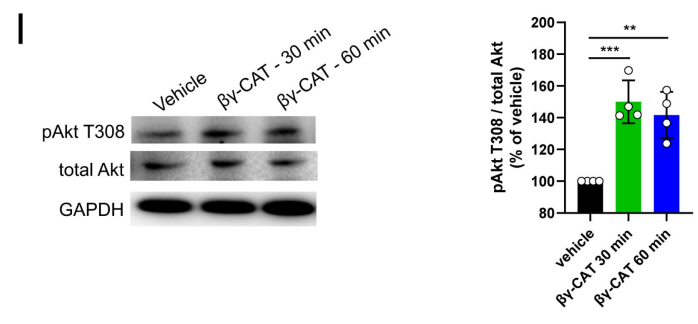

Fig. 3. $\beta \gamma$-CAT promotes macropinocytosis. $(A)$ Ultrastructural localization of

$\beta \gamma$-CAT in toad skin and UB tissues as analyzed by IEM. Vehicle (rabbit IgG control).

Endocytic vesicles formed by macropinocytosis (black asterisks), and distribution of $\beta \gamma$-CAT on vesicles (red arrows) and intercellular spaces (red triangles). ( $B, C)$

Immunedepletion of endogenous $\beta \gamma$-CAT decreased macropinocytosis. Toad skin $(B)$ 
and UB $(C)$ epithelial cells were incubated with $50 \mu \mathrm{g} / \mathrm{mL}$ anti- $\beta \gamma$-CAT antibody to immunodeplete endogenous $\beta \gamma$-CAT for 30 minutes. The mean fluorescence intensity was determined by flow cytometry with $100 \mu \mathrm{g} / \mathrm{mL}$ of $70 \mathrm{kDa}$ FITC-label dextran and Lucifer Yellow (LY) for 30 minutes. Rabbit IgG (antibody control). Vehicle (antibody absent control). $(D, E)$ The addition of purified $\beta \gamma$-CAT augmented macropinocytosis. The mean fluorescence intensity of LY and FITC-label dextran in toad skin (d) and UB (e) epithelial cells was determined by flow cytometry with or without additional $100 \mathrm{nM}$ or $50 \mathrm{nM} \beta \gamma$-CAT, respectively. $(F)$ Fold changes of $\left[\mathrm{Na}^{+}\right]$in toad UB epithelial cells (left, $n=6$ ) and MDCK (right, $n=8$ ) with and without the addition of $50 \mathrm{nM}$ or $10 \mathrm{nM} \beta \gamma$-CAT for 3 hours, respectively. $(G, H)$ The effect of inhibitors on macropinocytosis induced by $\beta \gamma$-CAT. Toad UB epithelial cells $(G)$ and MDCK cells $(H)$ were incubated with and without $100 \mu \mathrm{M}$ EIPA or $20 \mu \mathrm{M}$ WORT for 1 hour. Then the cells were cultured with $100 \mu \mathrm{g} / \mathrm{mL}$ FITC-label dextran with $50 \mathrm{nM}$ (toad UB cells) or $10 \mathrm{nM}$ (MDCK cells) $\beta \gamma$-CAT for 30 minutes. (I) Akt phosphorylation in response to $10 \mathrm{nM} \beta \gamma$-CAT in MDCK cells for 30 or 60 minutes as determined by western blotting (left), and bands were semiquantified with ImageJ (right). The black dotted line refers to the blank control, and data represent the mean $\pm \mathrm{SD}$ of triplicate samples in $B-E, G, H$. Data represent the mean $\pm \mathrm{SD}$ of at least three independent replicates in $F$ and $I . * P<0.05, * * P<0.01, * * * P<0.001$ and $* * * * P<0.0001$ by the unpaired $t$ test. All data are representative of at least two independent experiments. See also Fig. S3. 
A

Nucleus
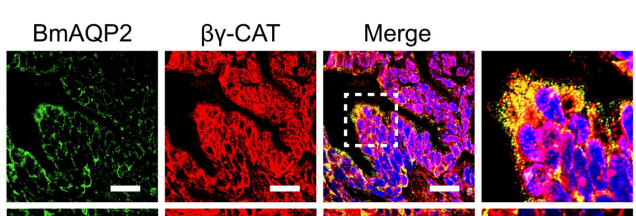

B
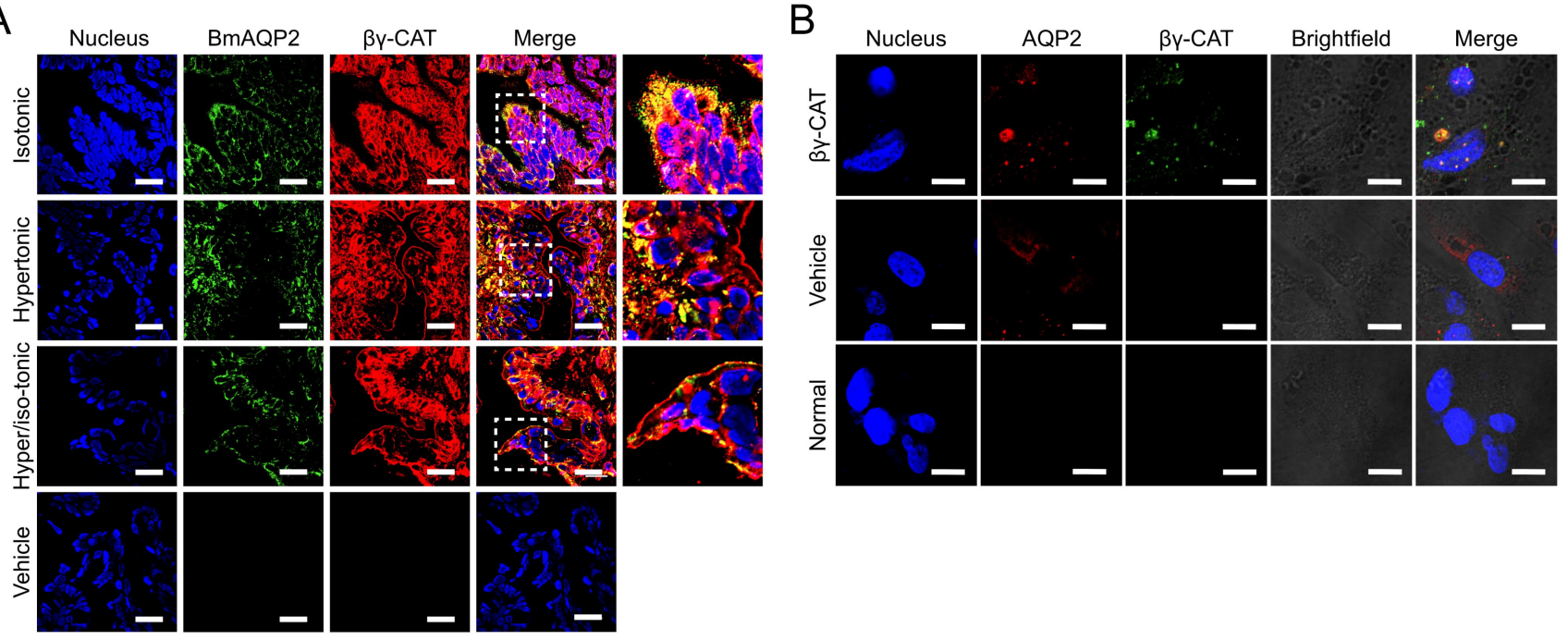

C

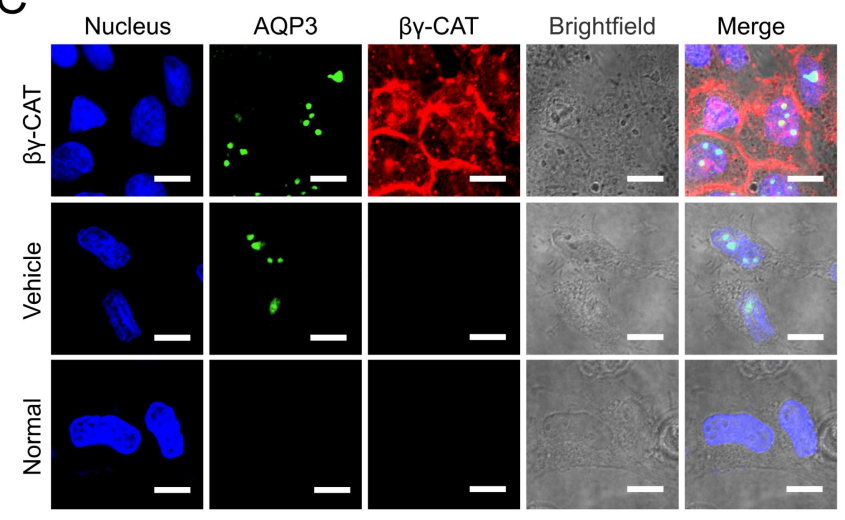

Fig. 4. $\beta \gamma$-CAT in AQP regulation. $(A)$ Colocalization of $\beta \gamma$-CAT and BmAQP2 in the UB tissue of B. maxima after the animals were placed in isotonic, hypertonic or hypertonic/isotonic Ringer's solution for 3 hours as analyzed by immunohistofluorescence (Scale bars, $25 \mu \mathrm{m})$. (B, $C$ ) Intracellular colocalization of $\beta \gamma$-CAT and AQP2 $(B)$ or AQP3 $(C)$ in MDCK cells with or without the treatment of $10 \mathrm{nM} \beta \gamma$-CAT for 15 minutes as determined by immunofluorescence (Scale bars, 10 $\mu \mathrm{m})$. All data are representative of at least two independent experiments. 
A

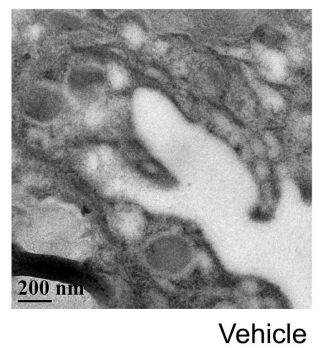

C

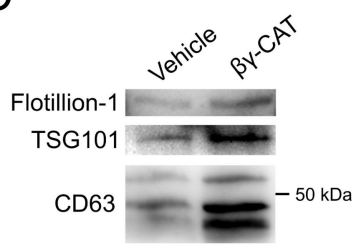

$\mathrm{F}$

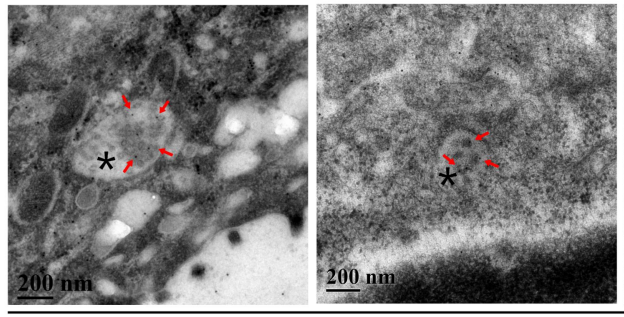

$\mathrm{D}$

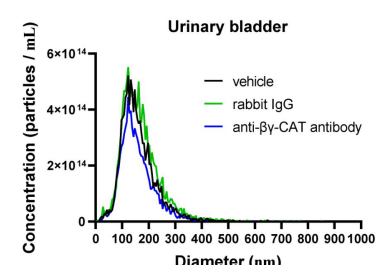

Diameter (nm)
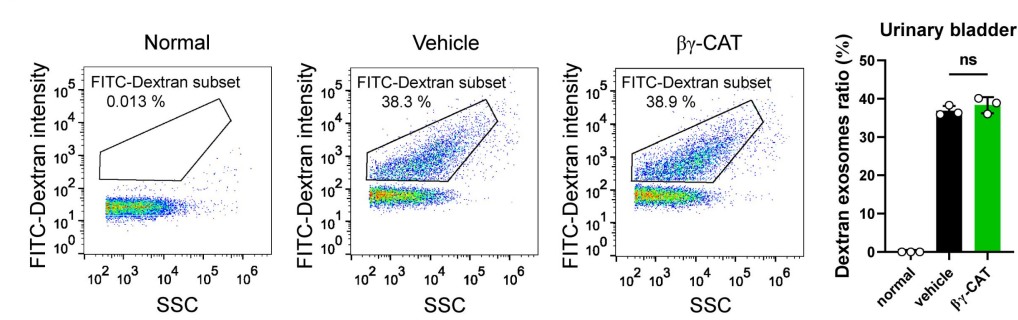

B
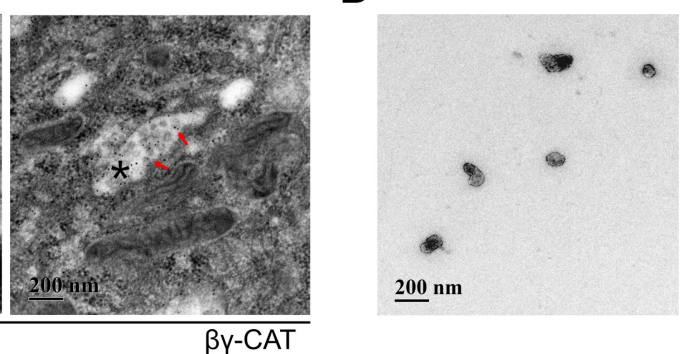

$E$
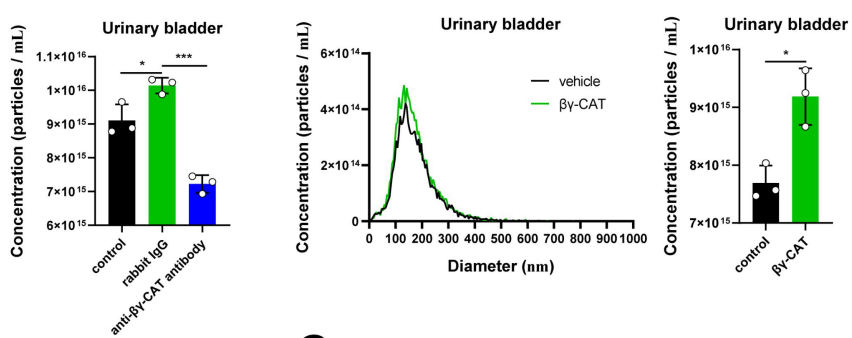

G

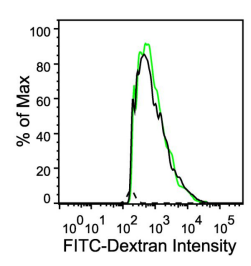

$\mathrm{H}$

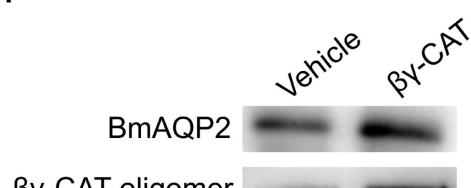

I

By-CAT oligomer
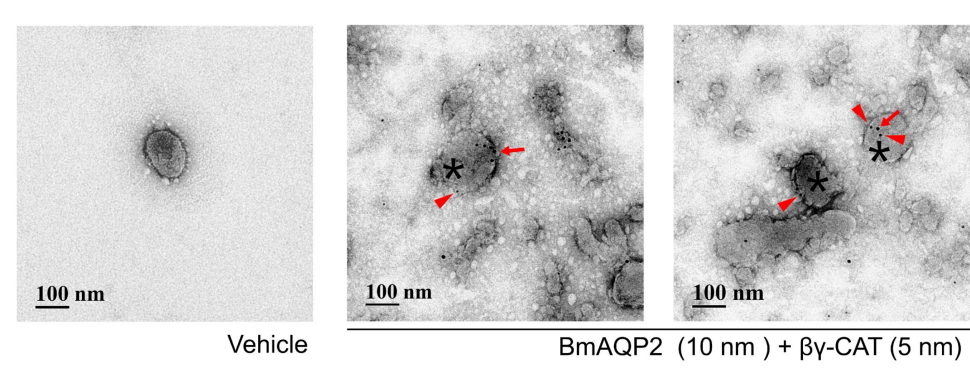

$\mathrm{J}$

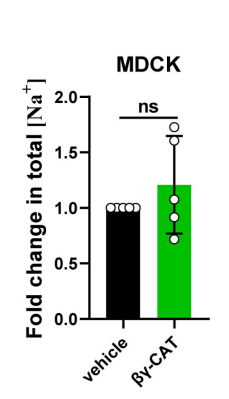

$\mathrm{K}$

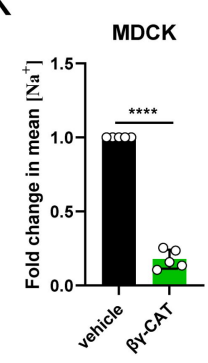

$\mathrm{L}$

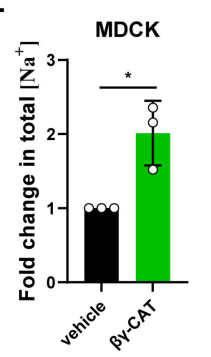

Fig. 5. $\beta \gamma$-CAT enhances exosome release. (A) Ultrastructural localization of $\beta \gamma$-CAT in toad UB tissue by IEM. $\beta \gamma$-CAT (arrow) was readily detected in MVBs (asterisk) or ILVs. Vehicle (rabbit IgG control). (B) TEM analysis of exosomes from the supernatant of toad UB epithelial cells cultured in vitro for 3 hours. (C) Western blotting analysis of flotillin-1, TSG101 and CD63 molecules in exosomes isolated from the supernatant of toad UB epithelial cells cultured in vitro for 3 hours with or 
without the addition of $50 \mathrm{nM} \beta \gamma$-CAT. $(D, E)$ Analysis of concentration and particle size (30-200 nm) of exosomes from toad UB epithelial cells with and without 50 $\mu \mathrm{g} / \mathrm{mL}$ anti- $\beta \gamma$-CAT antibodies $(D)$ or the addition of $50 \mathrm{nM} \beta \gamma$-CAT $(E)$ by NTA. $(F$, $G)$ The percentage $(F)$ and mean fluorescence intensity $(G)$ of dextran-containing exosomes from toad UB epithelial cells in a culture medium containing $1 \mathrm{mg} / \mathrm{mL}$ FITC-label dextran by Nanoflow Cytometry with or without the addition of $50 \mathrm{nM}$ $\beta \gamma$-CAT. $(H)$ Western blotting analysis of $\beta \gamma$-CAT and BmAQP2 in exosomes of toad UB epithelial cells cultured in vitro for 3 hours with or without the addition of $50 \mathrm{nM}$ $\beta \gamma$-CAT. (I) IEM determination of $\beta \gamma$-CAT and BmAQP2 in exosomes (asterisk) from toad UB epithelial cells. BmAQP2 and $\beta \gamma$-CAT were labeled with 10-nm (arrow) and 5 -nm (triangle) colloidal gold particles, respectively. $(J, K)$ Fold changes of total $(J)$ and mean $(K) \mathrm{Na}^{+}$concentrations in exosomes from the same number of MDCK with or without $10 \mathrm{nM} \beta \gamma$-CAT for 3 hours. $(L)$ Fold changes of total $\mathrm{Na}^{+}$concentrations in exosomes from the same number of MDCK under the hypertonic medium with or without $10 \mathrm{nM} \beta \gamma$-CAT for 3 hours. The bars represent the mean $\pm \mathrm{SD}$ of triplicate samples in $D-G$. Data represent the mean \pm SD of at least three independent replicates in $J$-L. ns $(P \geq 0.05),{ }^{*} P<0.05,{ }^{* *} P<0.01$ and $* * * * P<0.0001$ by the unpaired $t$ test. All data are representative of at least two independent experiments. See also Fig. S4. 


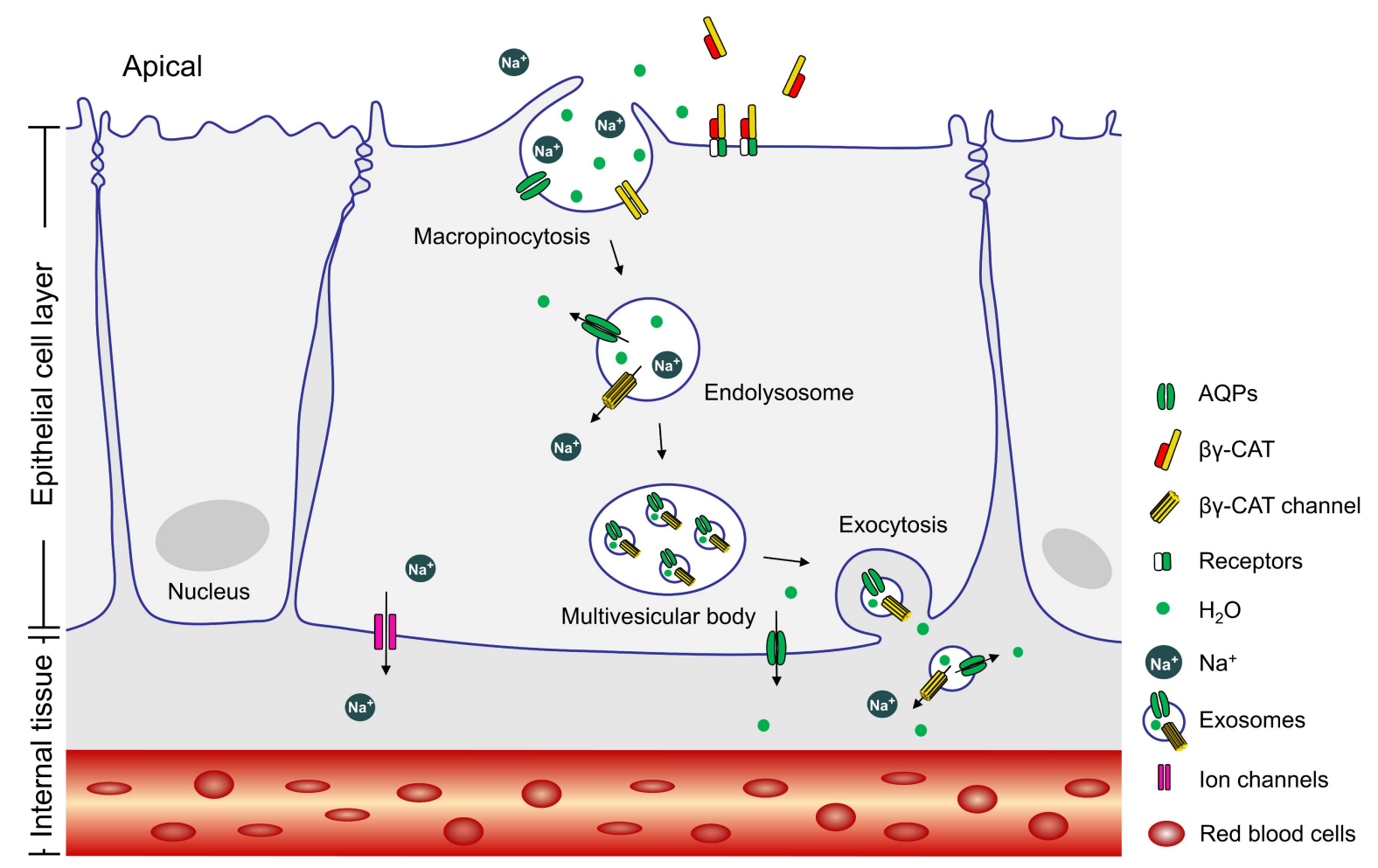

Fig 6. Proposed action model of $\beta \gamma$-CAT in water acquisition and maintaining.

$\beta \gamma$-CAT achieved intracellular vesicle transport and transcellular transport of water, $\mathrm{Na}^{+}$and AQP2 by promoting macropinocytosis (endocytosis) and exosome release (exocytosis). For a detailed description, see the test. Presentation of the epithelial cell layer and internal tissue is simplified. 